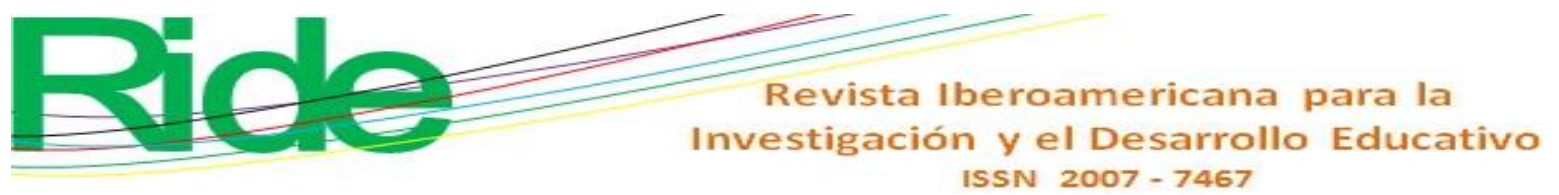

https://doi.org/10.23913/ride.v10i20.588

Artículos Científicos

\title{
Las disposiciones normativas de la participación social en una escuela primaria
}

\author{
The Normative Provisions of Social Participation in an Elementary School \\ As disposições normativas da participação social em uma escola primária
}

Felipe de Jesús Perales Mejía Universidad Pedagógica Nacional, Unidad 052, México

fperales_m@hotmail.com https://orcid.org/0000-0001-9051-546X

\section{Resumen}

El presente artículo expone los resultados de un estudio en caso, realizado en una escuela de educación primaria pública en México, a más de dos décadas de que el Gobierno federal impulsara una renovada participación social en educación con diversas normativas para las instituciones de educación básica. La perspectiva de la investigación fue cualitativa con orientación etnográfica. El propósito fue documentar la manera en que las disposiciones normativas son recontextualizadas por los agentes sociales. La investigación es coincidente con otras que han estudiado la cultura escolar; argumenta la necesidad de seguir documentando y recreando narrativamente lo que sucede en las instituciones educativas, tomando distancia de las propuestas elaboradas en otros contextos, con el fin de construir unas que se acerquen a los mundos de vida de los agentes sociales.

Palabras clave: educación básica, gestión, participación social. 


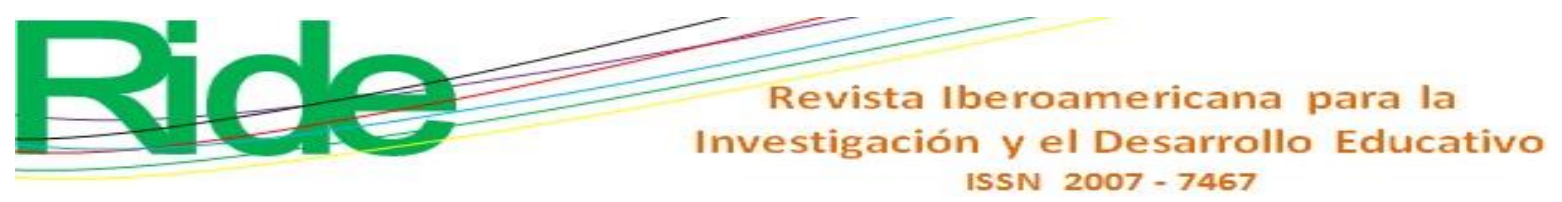

\section{Abstract}

This contribution has been a research made in an Elementary Pubic School, from Mexico. More than two decades after the Federal Government promoted a re-new social participation in the education, with various regulations for basic educational institutions. The research perspective, in which the study was conducted is the qualitative one, from an ethnographic perspective. In the text there have been re-built, in a narrative manner the way that the narrative regulations have been re-contextualized by the social agents.

This research has coincidence with others that have studied schools' cultures and this proposed the need of continue documenting and re- creating in a narrative way, what is going on in the educative institutions. Taking distance from elaborated projections from another contexts. In order to built one that reflects the real world of the social agents.

Keywords: basic education, management, social participation.

\section{Resumo}

Este artigo expõe os resultados de um estudo de caso realizado em uma escola pública pública no México, mais de duas décadas depois que o governo federal promoveu uma participação social renovada na educação com vários regulamentos para instituições de ensino básico. A perspectiva da pesquisa foi qualitativa, com orientação etnográfica. $\mathrm{O}$ objetivo era documentar a maneira pela qual as disposições normativas são recontextualizadas pelos agentes sociais. A pesquisa é consistente com outras pessoas que estudaram a cultura escolar; argumenta a necessidade de continuar documentando e recriando narrativamente o que acontece nas instituições de ensino, afastando-se das propostas feitas em outros contextos, a fim de construir aquelas que abordem o mundo da vida dos agentes sociais.

Palavras-chave: educação básica, gestão, participação social.

Fecha Recepción: Septiembre 2019

Fecha Aceptación: Diciembre 2019 


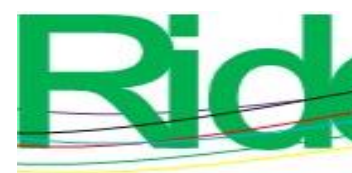

Revista Iberoamericana para la Investigación y el Desarrollo Educativo ISSN $2007-7467$

\section{Introducción}

La participación social es considerada por algunos investigadores como un intento de los gobiernos latinoamericanos de modernizar sus respectivos sistemas educativos. En esto los gobiernos estaban siguiendo las recomendaciones de organismos internacionales que se ocupan de asuntos técnicos y financieros. La adopción de las recomendaciones tenía como trasfondo la reconfiguración de las tradicionales relaciones del Estado con la sociedad, debido a la aparición de un nuevo actor en el proceso de globalización económica: el mercado (Torres, 2006).

En el sistema educativo mexicano, como en otros países latinoamericanos (Díaz, Alfaro, Calderón y Álvarez, 2010), la participación social en la educación fue uno de los ejes de la reforma educativa en la década de los 90, concretada con la firma del Acuerdo Nacional para la Modernización de la Educación Básica (Secretaría de Educación Pública [SEP], 1992). En la actualidad, se plantea como una necesidad para fortalecer la capacidad de gestión y autonomía en las escuelas (Diario Oficial de la Federación [DOF], 7 de marzo de 2014). Su finalidad es crear las condiciones para que alumnos, exalumnos, maestros, directivos, padres de familia y otros agentes sociales se involucren en la solución de los problemas que enfrentan las escuelas.

No obstante, la participación de los padres de familia en las escuelas tiene profundas raíces históricas; estos siempre han tomado parte en los asuntos escolares. Su participación inicia en el momento en que se institucionalizaron las escuelas primarias con la conformación de los sistemas educativos en el siglo XIX, llegando hasta la creación de asociaciones durante el siglo XX — como la Unión Nacional de Padres de Familia - para escuelas particulares católicas y por decreto presidencial del Estado mexicano, la Asociación Nacional de Padres de Familia (García, 2002).

En esta dimensión, los padres de familia han formado parte en la vida de las escuelas de manera regulada mediante diversos mecanismos, ${ }^{1}$ según sus intereses, necesidades, expectativas y cosmovisiones de lo que debe ser el proceso educativo. Las más de las veces su participación es inducida, seducida u obligada por las políticas que el Estado implementa, en la forma de cursos de acción implícitos o explícitos, buscando cumplir con las metas fijadas por los planes gubernamentales (Flores, 2011).

\footnotetext{
${ }^{1}$ Como la Confederación Nacional de Agrupaciones de Padres y Maestros, creada en 1929; el Reglamento para la Constitución y Funcionamiento de las Asociaciones de Padres de Familia en las escuelas dependientes de la SEP de 1949, o, en la historia reciente, el Reglamento de Asociaciones de Padres de Familia (DOF,4 de febrero de 1980).
} 


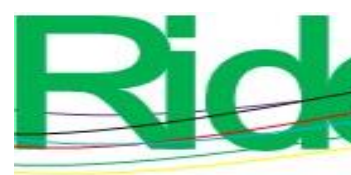

Revista Iberoamericana para la Investigación y el Desarrollo Educativo

ISSN $2007-7467$

La participación social, como estrategia y práctica del Gobierno, ha implicado implementar una serie de dispositivos normativos para las instituciones de educación básica. Algunos de estos son: la Ley General de Educación, en la que se demanda establecer Consejos de Participación Social (SEP, 1993); el Acuerdo 280, en el que se definen los lineamientos generales a los que se ajustarán la constitución y funcionamiento de los Consejos Escolares de Participación Social (SEP, 2000); el Acuerdo 535, en el que se precisan los lineamientos generales para su operación (SEP,2010), y el Acuerdo 717 (DOF, 2014), entre otros.

En este contexto histórico, el documento que se presenta tiene como objetivo presentar una aproximación narrativa sobre la manera en que las disposiciones normativas anteriormente enunciadas son concretadas por los agentes sociales — directora, padres de familia y profesoreshaciendo uso del sentido práctico en sus diversos modos de vida dentro de una escuela primaria (Bourdieu, 2002).

\section{Método}

El propósito de la investigación fue documentar la manera en que se concreta la participación social en una escuela primaria ubicada en la ciudad de Torreón, Coahuila, México. El estudio en caso se sitúa en la dimensión institucional y fue realizado durante dos ciclos escolares. Se enfoca en la particularidad de la generalidad social en la que son impulsadas las políticas de participación social en las escuelas de educación básica. Algunas de las preguntas que lo orientaron fueron: ¿Cómo participan docentes, directivos y padres de familia en la constitución de los Consejos de Participación Social? ¿Qué acciones realizan docentes y directivos en el ámbito administrativo para cumplir con las disposiciones de la SEP? En la escuela, en el tiempo en que se realizó la investigación, había un director, un subdirector liberado, 12 profesores, tres maestras de apoyo (inglés, educación artística y educación física) y dos intendentes. De los 12 profesores, ocho han cursado estudios de normal básica y de licenciatura en la Universidad Pedagógica Nacional; además, dos estudiaron la normal superior y dos están cursando una maestría.

La perspectiva con la que se realizó la investigación fue la cualitativa, con orientación etnográfica (Rockwell, 2009). Se utilizaron técnicas y recursos de investigación característicos de 


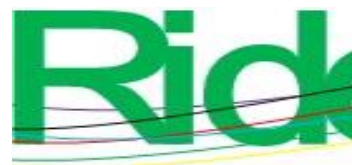

Revista Iberoamericana para la

Investigación y el Desarrollo Educativo

ISSN $2007-7467$

esta tradición: observación participante, entrevistas, encuestas, documentos del archivo de la institución y supervisión escolar. ${ }^{2}$

En un primer momento, el análisis de los registros de observación y entrevistas se realizó utilizando matrices descriptivas para identificar las categorías sociales (Coffey y Atkinson, 2003), que, como plexos de sentido, denotan los significados de los participantes. Posteriormente, se situaron en el entramado histórico en el que se promueve la participación social mediante dispositivos diversos (Bertely, 2000).

La documentación de cómo se involucran los actores escolares en la participación social permitió conocer lo que sucede en la escuela para reconstruir, a manera de correlato, la forma en que se están llevando a la práctica las disposiciones normativas establecidas para la constitución del consejo escolar.

La participación social en la escuela en la que se realizó el estudio se llevó a cabo con la presencia de algunos padres de familia que, de manera voluntaria u obligada, expresaron críticas y propuestas para enfrentar las dificultades que se presentan en la vida cotidiana de la escuela.

\section{Resultados}

Como lo prescribe el artículo 69 de la Ley General de Educación (SEP, 1993):

La autoridad escolar hará lo conducente para que en cada escuela pública de educación básica opere un consejo escolar de participación social, integrado con padres de familia y representantes de sus asociaciones, maestros y representantes de su organización sindical, quienes acudirán como representantes de los intereses laborales de los trabajadores, directivos de la escuela, exalumnos, así como con los demás miembros de la comunidad interesados en el desarrollo de la propia escuela (p. 28).

En el Acuerdo 535 se establecen las acciones para constituir los Consejos Escolares de Participación Social y fija el calendario de actividades de los miembros en cuatro sesiones y tres asambleas con la comunidad. Las actividades corresponden a lo que se denomina el ciclo funcional, que comprende tres etapas: 1) conformación, 2) gestión y 3) transparencia y rendición de cuentas (SEP, 2010).

\footnotetext{
${ }^{2}$ En el correlato, las citas contextuales de la observación participante se identifican con las letras $R O$, enseguida el día, mes y año. Las entrevistas, con la letra $E$, el día, mes y año.
} 


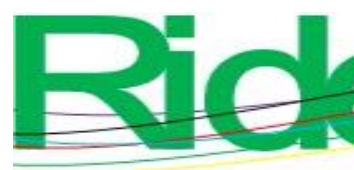

Revista Iberoamericana para la Investigación y el Desarrollo Educativo

ISSN $2007-7467$

Para la conformación de los consejos, las disposiciones fueron enviadas a los planteles a través de la estructura administrativa de la Secretaría de Educación en el Estado, como es tradición, de forma jerárquica: coordinación de nivel - jefatura de sector - supervisión - dirección escolar.

La dirección de la escuela es la instancia autorizada para convocar y dar cumplimiento a las disposiciones: integrar la mesa directiva de la Asociación de Padres de Familia ${ }^{3}$ y el Consejo Escolar de Participación Social. Sin embargo, la directora por lo regular encuentra dificultades para conformar ambas instancias, sobre todo porque implica la asistencia de padres y madres de familia. "La Sociedad de Padres de Familia tiene poca funcionalidad. Siempre es lo mismo: hay dificultad para conformar la mesa directiva porque nadie quiere participar”(E-090611).

Conforme los resultados de una encuesta, la escasa participación de los padres de familia se debe a que en la hora en que se realizan las reuniones, tanto del Consejo Escolar de Participación Social como de la Asociación de Padres de Familia, se encuentran laborando, y una falta en el trabajo significa una reducción de su salario. Por esa razón, en las reuniones se observa la asistencia de entre 35 y 40 padres de familia de un total de 200. Independientemente de la información previa que deben de tener para participar de manera informada en las asambleas escolares.

En la escuela en la que se realizó el estudio, en las reuniones que se celebraron para conformar tanto la Asociación de Padres de Familia como el Consejo Escolar de Participación Social, la directora se hacía acompañar de algunos miembros de ambos órganos escolares. Iniciaba la reunión con la exposición de los motivos de la reunión y la importancia de que los padres de familia participaran. En la reunión para la conformación del Consejo Escolar, la directora explicó las funciones que los integrantes asumirían en ese órgano escolar, las problemáticas que enfrentaba la escuela y enfatizó la necesidad de que participaran con propuestas o autopropuestas para su integración.

Sin embargo, al percibir que los padres de familia no tenían la intención de ocupar algún cargo, siguió la reunión con una actitud optimista a fin de lograr comprometer, de manera persuasiva, a los asistentes. Llegado a este punto se debe de tener en cuenta lo puesto a continuación, conforme lo establece el Acuerdo 280, en el artículo 20 (SEP, 2000):

\footnotetext{
${ }^{3}$ La elección, según el artículo 11 del citado reglamento, deberá ser durante los primeros 15 días del inicio del ciclo escolar; posteriormente se deberán organizar sesiones ordinarias cada dos meses, y extraordinarias cuando sean convocados los integrantes por el presidente. El artículo 29 establece los puestos unipersonales de presidente, vicepresidente, secretario, tesorero y seis vocales, en total 10 (DOF, 4 de febrero de 1980).
} 


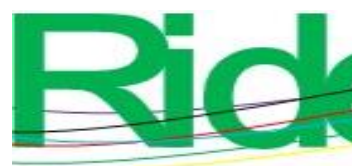

\section{Revista Iberoamericana para la Investigación y el Desarrollo Educativo \\ ISSN $2007-7467$}

Los consejos escolares de participación social en la educación se conformarán por un consejero presidente y hasta por quince consejeros. Se designará a un secretario técnico, que será nombrado por mayoría de votos de entre los integrantes de cada consejo escolar (p. 4).

Así, 17 personas, que sumadas a las 10 de la Asociación de Padres de Familia, suman un total de 27 miembros que integran, en teoría, los órganos escolares. Por lo tanto, no es poco el esfuerzo que debió hacer la directora para lograr la participación de los padres de familia.

La formación del Consejo Escolar de Participación Social busca la manera de contar con alguna ayuda, algún beneficio. Aquí hay quienes pueden hacerlo. No quiero decir quiénes, pero ya las estoy viendo... ¡Ándenle! ¡Anímense! ¿Alguien dice yo? Si no, vamos a tener que hacerlo como voluntario a fuerzas. Usted, señora, ¿nos ayudaría? (RO-230911).

En un primer momento, la directora informó a los padres de familia sobre los beneficios que se obtienen al cumplir administrativamente con la integración del Consejo Escolar de Participación Social, como recibir los apoyos que la SEP y el Gobierno del Estado proporcionan ("Escuelas al 100", "Desayunos Escolares", etc.). Sin embargo, su discurso no logró convencer a los asistentes para que se autopropusieran o propusieran como candidatos a ocupar algún cargo en el Consejo, por lo que designó de manera directa a las personas con las que había conversado previamente.

Una vez que se conformó el Consejo Escolar, solicitó a los integrantes que firmaran el formato del acta constitutiva para cumplir en tiempo y forma con el requisito administrativo. Posteriormente, invitó a los asistentes para que se integraran a los diferentes comités de alimentación, seguridad, lectura, etc., con la finalidad de atender las necesidades y prioridades de los alumnos y de infraestructura de la escuela. Hay que precisar que, durante ese ciclo escolar, la directora fungió como Consejera Presidente, de tal manera que las propuestas que los demás integrantes realizaran necesitarían contar con su autorización.

En esta dimensión, la constitución del Consejo Escolar de Participación Social en la escuela en cuestión es de tipo administrativo más que participativo; se cumple en tiempo y forma con las disposiciones que emiten las autoridades escolares, sin que los padres de familia se involucren de manera voluntaria. 


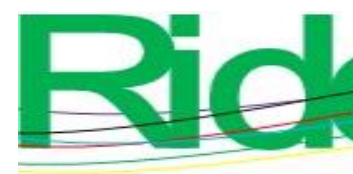

Revista Iberoamericana para la

Investigación y el Desarrollo Educativo

ISSN $2007-7467$

\section{Doble representación}

Según consta en las actas del ciclo escolar 2012-2013, del mes de septiembre, los mismos padres de familia ocuparon en $50 \%$ los cargos tanto de la Asociación de Padres de Familia como del Consejo Escolar de Participación Social. Es decir, había una doble representación en los dos órganos escolares, porque participar en la escuela requiere tiempo y la manera práctica de hacerlo de la directora es plantear soluciones inmediatas para que las autoridades educativas no la sancionen, pero también para evitar posibles conflictos entre los integrantes de ambos órganos escolares, independientemente de que no todos los padres de familia participen, solo unos cuantos.

\section{Alimentación saludable}

Con la finalidad de que los profesores cuenten con un apoyo económico que les ayude en los gastos que realizan en el desarrollo de las actividades escolares, existe un estanquillo en el que se venden algunos productos y las ganancias que se obtienen se comparten con la dirección escolar para solventar las necesidades que se generan en la escuela (E-080911).

Los artículos que se ofertan en el estanquillo no reflejan lo que promueven las campañas nacionales implementadas por la SEP para una alimentación saludable. Los artículos que se venden son frituras, golosinas y refrescos. Podemos notar cómo las campañas no solo tienen que enfrentarse a los hábitos alimenticios de la población en general, arraigados ya en la misma cultura, sino también al mismo personal docente de la escuela. El criterio que orienta la elección de los productos para la tiendita es la de tener mayores ventas y, por lo tanto, mayores ganancias. Esto es lógico si se piensa que los dividendos se reparten entre los docentes y la dirección del plantel para enfrentar diversas necesidades.

Sin embargo, como en la escuela se organizó el Comité del Consejo Escolar para promover la alimentación saludable, se constituyó un menú alternativo y este se ofreció salón por salón, con un costo de diez pesos a los alumnos que se interesaran en comprarlo (RO-081012).

Cuando los padres de familia ofrecieron productos saludables, las ventas de los otros alimentos descendieron. Por tanto, los ingresos de los docentes y de los directivos también disminuyeron. Sin embargo, esto no fue preocupante, ya que la directora apoyó la propuesta y estuvo dispuesta a permitir a los padres de familia para que vendieran sus productos en los salones en horas de clase, aun cuando este tipo de actividad sería un distractor para los alumnos. Lo anterior no fue de importancia para profesores, directivos ni para los padres de familia. 


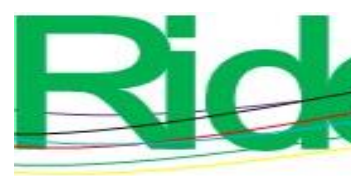

Revista Iberoamericana para la Investigación y el Desarrollo Educativo

ISSN $2007-7467$

Otros aspectos del "interés desinteresado" es la atención a los problemas de salud de los alumnos. Por ejemplo, la dirección autoriza la visita de representantes de instituciones del sector salud para que identifiquen a los alumnos que presentan problemas de sobrepeso y obesidad o citan a los padres de familia para que contesten encuestas.

Lo anterior, para el observador que es ajeno a la vida cotidiana de la escuela, puede resultar contradictorio porque, por un lado, los padres son convocados por los representantes del sector salud (apoyados por la directora) para obtener información acerca de su estilo de vida y alimentación. Por otro, durante los recreos, se permite a los alumnos consumir productos que difícilmente los ayudarán a controlar su peso. Sin embargo, la vida cotidiana en las escuelas y aulas, como lo documentaron Philip W. Jackson (1992) y Francois Dubet y Danilo Martuccelli (1998), tiene su propia lógica, la mayoría de las veces no escrita en el currículo que prescribe la administración, sino que adquiere su sentido práctico, basado en las contingencias e intereses de los agentes sociales.

\section{Discusión}

Hace más de dos décadas que el Estado impulsó una renovación de la participación social en la educación básica. Para ello, creó instancias colegiadas y promulgó leyes, acuerdos y lineamientos con el fin de transformar la subjetividad de los agentes sociales y sus prácticas. Según lo documentado en el presente estudio, los agentes sociales no se orientan siguiendo puntualmente las acciones prescritas, sino mediante el sentido práctico (Bourdieu, 1988, 2002), que no siempre refleja la propuesta de lo que el Estado busca con las leyes, los acuerdos y los lineamientos, sino la contingencia y los intereses de los actores sociales.

El estudio es coincidente con otros que han estudiado la cultura escolar y revela la necesidad de seguir documentando y recreando narrativamente lo que sucede en las instituciones educativas (Canales, 2006; Estrada, 2009; Perales, 2013, 2014 y 2015) para comprender el sistema de relaciones que las orienta. Asimismo, como señala Ezpeleta (2004), la escuela es una institución permeada por múltiples procesos en el que las innovaciones educativas implementadas desde la racionalidad técnica se circunscriben a sus propias lógicas, sin considerar otros procesos que permitan su articulación. 


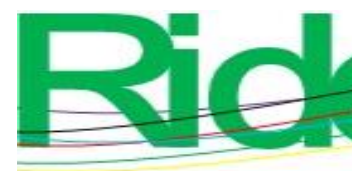

Revista Iberoamericana para la Investigación y el Desarrollo Educativo

ISSN $2007-7467$

\section{Conclusiones}

Desde el sentido, práctico y las razones que lo orientan, es posible comprender el doble juego de los representantes de la Asociación de Padres de Familia y del Consejo Escolar de Participación Social. Se comprende el "interés desinteresado" por acceder, permanecer y estar en la escuela con un estatus particular. Se percibe también la manera práctica en que la directora pretende controlar a los integrantes de ambos organismos. Finalmente, se alcanzan a comprender los intereses cruzados en la administración de la tiendita escolar y la manera en que se ofrece el menú con alimentos saludables a los alumnos, aun a costa de invadir los salones de clase y quitar tiempo destinado a la enseñanza.

Una de las fortalezas del presente estudio es el dar cuenta de la forma en que las disposiciones normativas se concretan en un determinado contexto escolar y social mediante razones prácticas de los agentes sociales, atravesado por múltiples procesos e intereses, diferentes al del contexto en que son diseñadas, ideadas, fantaseadas y anheladas como algo que se espera que suceda en las escuelas para transformar las prácticas y la cultura de los agentes sociales. Tal vez pueda compartir patrones similares en la manera en que se constituyen los Consejos Escolares de Participación Social en otros centros escolares, en el tiempo y los formatos que establecen las autoridades educativas, con el propósito de cumplir con las disposiciones administrativas, quedando lejos la implicación de los participantes porque las razones prácticas orientan el sentido de sus actuaciones. Sin embargo, muestra la particularidad de la generalidad social en la manera en que se implementan las políticas y dispositivos que pretenden impulsar la participación social.

Por otro lado, la limitación de este estudio es la imposibilidad de generalizar los resultados como una muestra representativa de lo que sucede en otras escuelas, porque es un estudio en caso. Es decir, solo una evidencia que muestra las condiciones de posibilidad en que se materializan las decisiones técnicas que pretenden modificar la cultura de la participación social.

Por lo anterior, es necesario analizar los procesos de recontextualización en el que se concretan, tomando en cuenta los intereses y el sentido práctico de los agentes implicados que se aceptan como normales al formar parte de la vida cotidiana de las instituciones.

Lo documentado tal vez contribuya a que los lectores, tomadores de decisiones y autoridades educativas tomen distancia para reconsiderar las propuestas de los organismos internacionales que se ocupan de asuntos técnicos y financieros, desde la racionalidad técnica, para construir otras que toquen más a los agentes sociales y sus mundos de vida, que se orientan por razones prácticas más que por disposiciones normativas, constituyendo subjetividades 


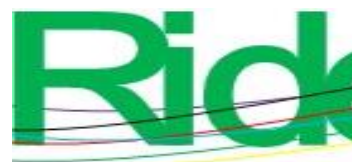

Revista Iberoamericana para la

Investigación y el Desarrollo Educativo

ISSN $2007-7467$

participativas que hagan posible la construcción de una ciudadanía comprometida en los procesos de formación.

\section{Referencias}

Bertely, M. (2000) Conociendo nuestras escuelas. México: Paidós.

Bourdieu, P. (1988) Cosas dichas. Buenos Aires, Argentina: Gedisa.

Bourdieu, P. (2002). Razones prácticas. Sobre la teoría de la acción. Barcelona, España: Anagrama.

Canales, S. A. (2006). La participación social en educación: un dilema por resolver. Perfiles Educativos, 28(113), 62-80. $\quad$ Recuperado de http://www.redalyc.org/pdf/132/13211304.pdf.

Coffey, A. y Atkinson, P. (2003). Encontrarle sentido a los datos cualitativos. Estrategias complementarias de investigación. Colombia: Editorial Universidad de Antioquía.

Díaz, B. C., Alfaro, P. B., Calderón, A. y Álvarez, L. N. (2010). Los protagonistas de los Consejos Educativos Institucionales: Tensiones y Dilemas de la Participación en la Gestión de la Escuela Pública. Revista iberoamericana sobre Calidad, Eficiencia y Cambio en Educación. $\quad 8(3), \quad 31-49 . \quad$ Recuperado de http://www.rinace.net/reice/numeros/vol8num3.htm.

Dubet, F y Martuccelli, D. (1998). En la escuela. Sociología de la experiencia escolar. España: Losada.

Diario Oficial de la Federación [DOF]. (4 de febrero de 1980). Reglamento de Asociaciones de Padres de Familia. Diario Oficial de la Federación. Recuperado de http://dof.gob.mx/nota_detalle.php?codigo=4851086\&fecha=02/04/1980.

Diario Oficial de la Federación [DOF]. (7 de marzo de 2014). Acuerdo 717, por el que se emiten los lineamientos para formular los programas de Gestión Escolar. Diario Oficial de la Federación.

Estrada, M. (2008). La participación social en educación: Hacia una comunidad escolar en Las Margaritas, Chiapas. Revista Interamericana de Educación para la Democracia, 1(2), 186204. Recuperado de http://www.dhl.hegoa.ehu.es/ficheros/0000/0529/Participaci.pdf.

Ezpeleta, M. (2004). Innovaciones educativas. Reflexiones sobre los contextos en su implementación. Revista Mexicana de Investigación Educativa, 9(21), 403-424. 


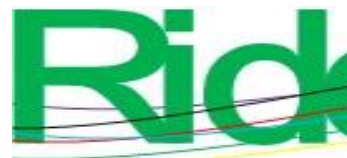

Recuperado
Revista Iberoamericana para la

Investigación y el Desarrollo Educativo

ISSN $2007-7467$

https://www.comie.org.mx/revista/v2018/rmie/index.php/nrmie/article/view/853/853.

Flores, P. (2011) Análisis de política educativa. Un nuevo impulso. Revista Mexicana de Investigación Educativa, 16(50), 687-698. Recuperado de https://www.comie.org.mx/revista/v2018/rmie/index.php/nrmie/article/view/390/39 0 .

García, A. (2002), La participación de los padres de familia en educación, siglos XIX y XX. En Galván, L. E. (coord. $\left.{ }^{a}\right)$, Directorio de historia de la educación en México. Recuperado de http://biblioweb.tic.unam.mx/diccionario/htm/articulos/sec_9.htm

Jackson, W. P. (1992). La vida en las aulas. Madrid, España: Morata.

Perales, M. F. (2013). Disposiciones y Posiciones en la Participación Social. Ponencia presentada en el XII Congreso Nacional de Investigación Educativa. Guanajuato, del 18 al 22 de noviembre de 2013. Recuperado de http://www.comie.org.mx/congreso/memoriaelectronica/v12/doc/0092.pdf.

Perales, M. F. (2014). La participación social en educación: entre el habitus comunitario y la obligación administrativa. Cpu-e Revista de Investigación Educativa, 8, 86-119. Recuperado de http://cpue.uv.mx/index.php/cpue/article/view/967.

Perales, M. F. (2015). Tensiones de la Participación Social en una Escuela Primaria. Ponencia presentada en el XIII Congreso Nacional de Investigación Educativa. Chihuahua, del 16 al 20 de noviembre de 2015, Recuperado de http://www.comie.org.mx/congreso/memoriaelectronica/v13/doc/1796.pdf.

Rockwell, E. (2009). La experiencia etnográfica. Historia y cultura en los procesos educativos. Buenos Aires, Argentina: Paidós.

Secretaría de Educación Pública [SEP]. (1992). Acuerdo Nacional para la Modernización de la Educación Básica. México: Secretaría de Educación Pública.

Secretaría de Educación Pública [SEP]. (1993). Ley General de Educación. México: Secretaría de Educación Pública. 


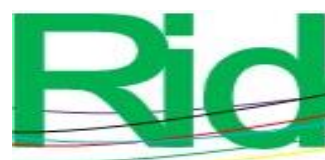

\section{Revista Iberoamericana para la Investigación y el Desarrollo Educativo ISSN $2007-7467$}

Secretaría de Educación Pública [SEP]. (2000). Acuerdo 280 por el que se establecen los Lineamientos Generales a los que se ajustarán la constitución y el funcionamiento de los Consejos de Participación Social en la Educación. México: Secretaría de Educación Pública. Recuperado de http://normatecainterna.sep.gob.mx/work/models/normateca/Resource/248/1/images /acuerdo_280_lineamientos_constitucion_funcionamiento_participacion_social.pdf. Secretaría de Educación Pública [SEP]. (2010). Acuerdo 535 por el que se emiten los Lineamientos Generales para la Operación de los Consejos Escolares de Participación Social. Diario Oficial de la Federación.

Torres, R. M. (2006). Participación ciudadana y educación: Una mirada amplia y 20 experiencias en América Latina. Punta del Este, Uruguay: Unidad de Desarrollo Social y Educación (UDSE) de la OEA. 